\title{
Reliability of an experimental method to analyse the impact point on a golf ball during putting
}

Ashley K. Richardson, Andrew C. S. Mitchell and Gerwyn Hughes

This is an Accepted Manuscript of an article published by Taylor \& Francis in Sports Biomechanics on 23rd June 2015, available online: https://dx.doi.org/10.1080/14763141.2015.1052542

Richardson, A.K., Mitchell, A.C.S. and Hughes, G. 2015. Reliability of an experimental method to analyse the impact point on a golf ball during putting. Sports Biomechanics. 14(2): pp.206-215. doi: 10.1080/14763141.2015.1052542 
1 Reliability of an experimental method to analyse the impact point on a golf ball 2 during putting.

3

$4 \quad$ Ashley K. Richardson ${ }^{1}$, Andrew C. S. Mitchell ${ }^{2}$, \& Gerwyn Hughes ${ }^{3}$

$5{ }^{1}$ Division of Sport and Exercise Sciences, School of Social and Health Sciences,

6 Abertay University, UK. ${ }^{2}$ Department of Sport Science and Physical Activity, Faculty

7 of Education and Sport, University of Bedfordshire, UK. ${ }^{3}$ Sport, Health and Exercise

8 Subject Group, School of Life and Medical Sciences, University of Hertfordshire, UK.

9

10 KEYWORDS: Biomechanics, reliability, golf putting, kinematics.

11

12

13

14

15

16

17

18

19

20

21

22

23

24

25 


\section{Abstract}

This study aimed to examine the reliability of an experimental method identifying the location of the impact point on a golf ball during putting. Forty trials were completed using a mechanical putting robot set to reproduce a putt of $3.2 \mathrm{~m}$, with four different putter-ball combinations. After locating the centre of the dimple pattern (centroid) the following variables were tested; distance of the impact point from the centroid, angle of the impact point from the centroid and distance of the impact point from the centroid derived from the $X, Y$ coordinates. Good to excellent reliability was demonstrated in all impact variables reflected in very strong relative (ICC $=0.98-$ 1.00) and absolute reliability (SEM\% $=0.9-4.3 \%)$. The highest SEM\% observed was $7 \%$ for the angle of the impact point from the centroid. In conclusion the experimental method was shown to be reliable at locating the centroid location of a golf ball, therefore allowing for the identification of the point of impact with the putter head. Therefore is suitable for use in subsequent studies.

Words: 174

\section{Introduction}

Putting accounts for $43 \%$ of shots made in golf (Pelz, 2000). Despite a number of

44 studies having identified a positive correlation between successful putting performance and overall score (Dorsel \& Rotunda, 2001; Quinn, 2006; Wiseman \& Chatterjee, 2006) there is still a lack of understanding of the elements that constitute a successful golf putt. Green reading (selecting correct initial ball direction), aim 48 (placing putter face square to selected line), stroke and ball roll are the main biomechanical factors considered to contribute to a successful putt (Karlsen, Smith 
and Nilsson, 2008). One variable that has not been analysed extensively within the

51 literature is the impact point on the golf ball.

Literature investigating the effect of impact point on the resulting kinematics of the golf ball during putting is limited. Cross and Nathan (2007) reported the gear effect

55 chipping. Raising the question as to whether dimple design negatively affects putting.

(the rotation of the moving object around its centre of mass due to an off-axis impact) in ball collisions, including the golf ball. Results demonstrated the rate of spin increased when the angle of incidence (degree of deviation away from a perpendicular collision) is increased (Cross \& Nathan, 2007), which could potentially be detrimental to putting performance by increasing the variability associated with the resultant putt. Cross and Nathan (2007) concluded that the gear effect occurs as a result of static friction between the ball and object during a collision. A clear limitation of the Cross and Nathan (2007) study is that during the experimental protocol, the ball was collided off a wooden block which is not as appropriate as the use of a putter. Alessandri (1995), Lorensen and Yamrom (1992), and Penner (2002) have all proposed mathematical models of the motion of a putted golf ball over the surface of the green.

More research is required to examine whether the impact point during the putter face - ball interaction influences the success of the subsequent putt. Additionally, many ball manufacturers choose not to include any performance information regarding putting, with predominant focus on driving distance and 'soft' feel during pitching and 3 
74 Currently no studies have investigated how variation in the impact point on the golf

75 ball influences the resulting kinematics of the golf ball and, furthermore, how different dimple patterns on the ball can affect the kinematic variables of the shot. No method for the analysis of the effect of the impact point has been devised or suggested within the literature. Therefore, the aim of this study was to develop and assess the reliability of a method of locating a centroid location and identifying the impact point on a golf ball. If found to be reliable, it will allow for the method to be adopted and used in further research, such as determining whether the impact point on a golf ball has an effect on the resultant kinematics of the ball during the golf putt. It was hypothesised that the method of locating a centroid location and the two methods of identifying the impact point on a golf ball would be reliable.

\section{Methods}

\section{Experimental set - up}

All testing was completed on an artificial putting surface (Huxley Golf., Hampshire, UK) $(3.66 \times 4.27 \mathrm{~m}$ ) registering 12 on the stimpmeter (The United States Golf Association., NJ, USA). A stimpmeter is a device used to measure green speed (initial ball velocity $=1.83 \mathrm{~m} / \mathrm{s}$, ball travelled $3.65 \mathrm{~m}$ ). A mechanical putting arm mounted on an $360 \mathrm{~kg}$ bearing was set up to simulate a level $3.2 \mathrm{~m}$ putt, with a square to square swing path to ensure a square club face at impact. This refers to a single horizontal axis perpendicular to the putting line.

Two putters with different putter face characteristics (grooved or non grooved) were selected and used for the experiment. The GEL ${ }^{\circledR}$ (GEL GOLF., Wan Chai, Hong 
Kong) Vicis putter (grooved face) had a $69^{\circ}$ lie (angle formed by the shaft and sole of the putter head when the putter is in a neutral position) and $2.5^{\circ}$ loft (angle formed by the putter face and level surface when the putter is in a neutral position), and the Odyssey (Callaway Golf Europe Ltd., Surrey, UK) White Hot \#3 (non-grooved) had a $69^{\circ}$ lie and $2.5^{\circ}$ loft. Srixon (Srixon Sports Europe LTD., Hampshire, UK) Z-STAR golf balls and Titleist (Acushnet Europe Ltd., Cambridgeshire, UK) Pro V1 golf balls were used in the protocol. These particular golf balls were chosen due to them being two popular balls on the market, similar in construction and both brands premium offerings.

The golf balls were aligned using two Superline (Property Perspective Ltd, Warwick, UK) two-dimensional (2D) line lasers fixed to a $360^{\circ}$ graduated base. One was placed directly behind the ball and the other was placed $90^{\circ}$ to the path of the golf ball intersecting a visual putting aid printed on the ball. This split the golf ball into four equal sections ensuring the same position of the ball for each trial. A Canon (Canon Europe Ltd, Uxbridge, UK) EOS 1000d camera was situated on a stationary tripod in front of the line of the golf putt $2.5 \mathrm{~m}$ away from impact.

\section{Procedure}

The first putter was held securely in the mechanical putting arm and aligned using a swing path laminate and laser line to ensure a square to square swing path. The counterbalanced putting arm block was set to produce a putt of $3.2 \mathrm{~m}$. The putting arm was attached to a weighted pole and released using an electromagnet to reduce friction to a minimum. Before the first trial was completed, a thin layer of pigmented emollient was applied to the face of the putter and smoothed out to confirm an even 
124 coating. This was repeated after every trial. The golf balls were aligned using the two

125 Superline 2D line lasers fixed to a $360^{\circ}$ graduated base as described in the 126 experimental set - up.

128 After each trial a picture was taken (Canon EOS $1000 \mathrm{~d}$ ) with the ball placed $5 \mathrm{~cm}$ to 129 the right of the original position before impact, angled to show the pigmented 130 emollient imprint on the ball and the imprint of the dimple pattern left on the putter 131 face. The ball was then cleaned of all pigmented emollient using an alcohol wipe and 132 the next trial was completed. Each putter-ball combination had a total of 20 trials 133 recorded (total 80 trials).

Data Processing

\section{Determining the centroid location}

137 Two 2D structures (Figure 1) were developed matching the Titleist and Srixon golf 138 ball dimple patterns using Microsoft PowerPoint 2011 to locate the centroid $(0,0$ 139 coordinate of the dimple pattern). The Srixon golf ball had a single consistent size of 140 dimple and therefore an equilateral triangle with a line drawn at every vertex fitted the 141 dimple pattern identifying the centroid ( 0,0 coordinate) of the three dimples (Figure 1 142 A). In contrast the Titleist golf ball had two sizes of dimple (Figure 1 B), one smaller 143 dimple encapsulated by 5 larger dimples, so a pentagon with a line drawn at every 144 vertex fitted the dimple pattern, identifying the centroid $(0,0$ coordinate) of the six 145 dimples. 


\section{Scaling the picture}

150 The photograph from each trial was exported into Adobe Photoshop CS5 (Adobe

151 Systems Incorporated., CA, USA) and scaled using the known length of the GEL ${ }^{\circledR}$ 152 and Odyssey putters hosel. The hosel was selected as it was flat on each of the 153 putters and therefore was the most appropriate part to measure accurately.

155 The Photoshop ruler tool was used to calculate the angle that the ball was placed at. 156 This was to confirm that the 2D structure was placed in the correct and same 157 position, giving the same centroid (0, 0 coordinate) for each trial.

159 Calculating the centre of the impact area

160 To calculate the centre of the impact area or the impact point, a polygon was drawn 161 at the four outermost edges of the impact area (Figure 2). The first edge was drawn 162 horizontally from the two outermost edges and the angle was adjusted to the angle of 163 the dimple pattern identified (Figure $2 \mathrm{~A}$ ) when superimposing the 2D structure on the

164 ball. This line was then copied and superimposed at the opposite outermost edge 165 (Figure 2 B). These steps were repeated for the two vertical lines (Figure 2 C and 2 166 D). Each side was parallel to the opposite side and adjusted to fit correctly together.

167 Generally this involved either lengthening or shortening the horizontal lines and this 168 allowed for the polygon to be intersected from its four corners (Figure $2 \mathrm{E}$ and $2 \mathrm{~F}$ ) 169 giving the centre point of the impact area.

171 [FIGURE TWO ABOUT HERE] 
173 The Photoshop ruler tool was then used to measure the distance and angle of the

174 impact point from the centroid of the dimple pattern, producing a measurable vector.

175 Zero degrees were directly north of centroid. Additionally, the $X$ and $Y$ coordinates

176 were measured from the centroid of the dimple pattern using vertical and horizontal

177 guides. Pythagoras' theorem $\left(x^{2}+y^{2}=z^{2}\right)$ was used to calculate the distance of the

178 centre of the impact area to the centroid location to provide an alternative

179 measurement technique to compare to the accuracy of the angle distance method.

180

181 Calculating the area of the impact zone

182 Scientific image processing software ImageJ (National Institutes of Health, Bethesda,

183 Maryland, USA) was used to calculate the surface area of the impact area. The 184 polygon selection tool was used to draw (at $0.5 \mathrm{~mm}$ intervals) around the impact area 185 imprint on the golf ball (Figure 3) and gave an output of the surface area.

[FIGURE THREE ABOUT HERE]

189 Each putter-ball combination was processed and then reprocessed 24 hours later under the same conditions without reference to the previous analysis to keep the 191 reliability testing blind.

\section{Data Analysis}

194 Data were exported to statistical software packages Microsoft Excel 2011 and SPSS 195 v19 (SPSS Inc, Chicago, USA) for analysis. Reliability was assessed for the 196 following variables: distance of the impact point from the centroid (distance from the 197 centroid to the centre of the impact zone), angle of the impact point from the centroid 
198 (the angle of the centre of the impact zone from the centroid), $X$ coordinate from the

199 centroid, $\mathrm{Y}$ coordinate from the centroid and the resultant distance from the centroid

200 (using the $X, Y$ coordinates and the following formula: $x^{2}+y^{2}=z^{2}$ ). To ensure

201 unbiased results, the test-retest analysis was completed blind, without reference to

202 the other days analyses.

203

204 The data were found to be normally distributed using a Shapiro - Wilk test for 205 normality. A combination of descriptive (mean \pm SD and change in mean $\pm 95 \%$ 206 confidence limits (CL) (expressed as a percentage) and reliability statistics were 207 used. The change in mean and 95\% CL stipulated an indication of absolute variation 208 between the data sets.

209

210 Reliability statistics were the standard error of measurement expressed as a 211 percentage (SEM\%) (formula: $S E M=S D \sqrt{1-I C C}$ ), a two - way mixed intraclass 212 coefficient (ICC) (formula: $\frac{1-S D^{\wedge} 2}{S D^{\wedge} 2}$ were used.) (Hopkins, 2000) and a Cohen's 213 repeated measures effect size (ES). The boundaries set for the coefficient statistics 214 were; $r=0.8-1.0$, very strong, $r=0.6-0.8$, strong, $r=0.4-0.6$, moderate, $r=0.2$ $215-0.4$, weak, $r=0.0-0.2$, no relationship (Salkind, 2011). In accordance with 216 Saunders, Pyne, Telford and Hawley (2006) ES were interpreted as < 0.1 as trivial, $2170.1-0.6$ as small, $0.6-1.2$ as moderate and $>1.2$ as large. Assessing these 218 statistics as a collective group will provide a clear impression of the relibiability and 219 reproducibility of the method. For a reliabiltiy rating of 'excellent' the criteria 220 threshold was change in mean $<5 \%$, ICC $>0.90$, SEM $\%<10 \%$ and ES $<0.60$. For 221 'good' reliability, all but one criteria had to be met, for 'moderate' reliability all but two 
222 criteria had to be met, and 'poor' reliability was defined as three of the criteria not

223 being met (Joseph, Bradshaw, Kemp \& Clark, 2013).

224

\section{Results}

\section{Overview of reliability}

227 Tables 1 to 4 present descriptive and reliability statistics for the impact varibles.

228 Reliability was catagorised as excellent for all combined putter-ball combinations for 229 each of the four impact variables. When putter-ball combinations are considered 230 separately, the lowest reliability category demonstrated was good (the only failed 231 criteria was the ES).

\section{Surface Area}

234 Surface area results (Table 1 ) noted excellent - good reliability catagories between 235 the four putter-ball combinations. The SEM\% for all four putter-ball combinations 236 between Test 1 and Test 2 were $<3.3 \%\left(<1 \mathrm{~mm}^{2}\right.$ when considered as a raw number) 237 and the ICC values demonstrated very strong reliability for the combined group and 238 individual putter golf ball combintations (ICC $=0.95-0.99)$. For the three putter-ball 239 combinations that were catagorised as demonstrating good reliability, the ES was the

240 criteria that was broken. The largest change in mean \% scores were observed for 241 the Odyssey-Srixon combination at 3.2\%. The 95\% CL was consistent across groups 242 ranging from $2.7-3.0 \%$. At first glance, this variance may look relatively large, 243 however, when considered with the change in mean percentage the largest variance 244 between means was $6.1 \%$. This does emphasise the fact that care is needed when 245 processing the images for surface area. 


\section{Distance of impact point from the centroid location}

250 The combined putter-ball combinations demonstrated excellent reliability for the 251 distance of the impact point from the centroid location impact variable, this was 252 apparent for the individual putter-ball combinations apart from the GEL ${ }^{\circledR}$-Titleist group 253 (Table 2). The ICC was consistently very strong $(I C C=1.00)$ and was coupled with 254 consistently low SEM\% values $(1.6-2.9 \%)$. The change in mean percentage were consistently low across all four combinations along with the $95 \% \mathrm{CL}$. Again, the ES was the failed criteria for the $\mathrm{GEL}^{\circledR}$-Titleist combination, catagorising the reliability as 257 good.

[TABLE TWO ABOUT HERE]

Distance of the impact point from the centroid derived from the $X, Y$ coordinates

263 Distance data derived from the $\mathrm{X}, \mathrm{Y}$ coordinates (Table 3) demonstrated excellent 264 reliability across the putter-ball combinations except from the $\mathrm{GEL}^{\circledR}$ - Srixon 265 combination which was catagorised as good (SEM\% $=1.6-3.2 \% ;$ ICC $=0.99-$ 266 1.00). Descriptive statistics reassert the excellent reliability demonstrated, no 267 irregularities were observed for any data set. The SD remained consistant for all 268 groups across all trials, suggesting the variability observed actually existed rather 269 than being an analysis error.

271 When comparing the two methods to measure the distance from the centroid location 
272 the distance (measured directly) and angle method had a SEM\% range of 1.6 -

$2732.9 \%$, when dirived from the $X, Y$ coordinates the SEM\% range was $1.6-3.2 \%$.

274 Therefore, the distance from the centroid when measured directly, demonstrated

275 marginally better absolute reliability, but when the differences in SEM\% are

276 insignificant, both methods can be considered reliable at measuring the impact point.

277 A general trend identified that the distance derived from the $\mathrm{X}, \mathrm{Y}$ coordinates were

278 slightly shorter than that when directly measuring the impact point from the centroid,

279 but the differences were minimal and did not increase as the distance from the

280 centroid increased. Therefore as long as one method is chosen and all trials are 281 analysed using the same procedure, both methods could be used to calculate the 282 distance of the impact point from the centroid.

283

284

[TABLE THREE ABOUT HERE]

285

286

\section{Angle of the impact point from the centroid location}

287 Reliability was categorised as excellent for all putter ball combinations except from 288 the Odyssey-Titleist combination which was catagorised as good (failing the ES criteria for inclusion to excellent reliability) (Table 4). This was particularly reflected in very low SEM\% $(0.9-4.3 \%)$ and very strong ICC scores $(0.98-1.00)$ showing very

291 strong relationships between Test 1 and Test 2. Descriptive statistics confirm very 292 strong reliability with no apparent anomalies for the combined data set or individual 293 putter-ball combinations, with consistent SD observed. 


\section{Discussion and Implications}

298 The aim of this study was to test the reliabilty of a method to identify the impact point on a golf ball. This would allow for further analysis to see the effect on resultant ball roll kinematics. It was hypothesised that the two methods calculating the distance and direction of the impact point from the centroid would be reliable, this can be

302 accepted. The methods were the manual measurement of the distance coupled with 303 the angle from the centroid location and measuring the $X, Y$ coordinates and 304 calculating the distance of the impact point from the centroid. The results for both 305 methods were reliable, therefore both methods are appropriate for future analysis. It 306 was the preference of the authors to use the distance angle measurement, over the $307 \mathrm{X}, \mathrm{Y}$ coordinates method. Additionally, this method allows for increased statistical 308 power during multiple regression analysis, due to reducing the number of 309 independent variables by one. Therefore this method can be considered suitable to 310 evaluate the effect of the impact point on the subsequent kinematics of the golf ball.

312 It is worthy noting that greater variability for angle from the centroid location (Table 4) 313 (as reflected in the SEM\%) was observed in the Srixon ball in comparison to the 314 Titleist ball when hit with the $\mathrm{GEL}^{\circledR}$ putter. This could potentially reveal that certain 315 styles of putters (grooved faced/traditional faced) demonstrate more consistency 316 when used in conjunction with certain brands of balls with differing dimple patterns.

318 It is difficult to draw comparisons to other methods that identify and analyse the 319 impact point on a golf ball, as currently within the literature the variable has been 320 overlooked. Research by Brouillette and Valade (2008), Brouillette (2010) and 321 Hurrion and Hurrion (2008) has been limited to analysis of the roll of the golf ball, with 
322 no discussion of the effect of the impact point. This is also apparent in studies 323 (Alessandrini 1995; Lorensen \& Yamrom, 1992 and Penner, 2002) that have used

324 mathematical models to predict the roll of the golf ball. Karlsen et al. (2008) state

325 that impact point accounts for 3\% of direction variability, however, they only tested 326 impact from the sweet spot in comparison to horizontal miss-hits and not the 327 variability observed within each impact type, therefore this claim may be 328 unsubstantiated.

330 A potential limitation of this study is that there is no obvious criterion measure that 331 this method can be compared to. Therefore the validity of this method cannot be 332 tested. Additionally, some researchers may demonstrate more subjective variability 333 and less accuracy (in undertaking the method to locate the centroid location and 334 subsequent impact point). To ensure reliability of future analysis using this method, it 335 is suggested that a pilot analysis is undertaken before the main analysis. This is to 336 certify that there is minimal variablilty during the data processing. By demonstrating 337 very strong relative and absolute reliability, it shows that in this study the researcher 338 was consistently accurate in identifying all variables.

\section{Conclusion}

341 Good to excellent reliability was demonstrated for all impact variables when the 342 reliabilty statistics were interpreted as a collective group during analysis of the 343 experimental method to determine the impact point of the putter on the golf ball. All 344 variables had very low SEM\% and demonstrated very strong relative reliability (ICC $345=0.95-1.00)$. This method can be considered reliable in the assessment of the 346 point of impact on the golf ball. Therefore, the method can be used for subsequent 
analysis of the effect of variation in the impact point on the golf ball on subsequent

348 ball roll kinematics. Care needs to be taken during the entire data processing

349 method, due to the high number of stages involved in the image processing protocol.

350 If an error is made during one stage it will ultimately effect the subsequent stages, 351 therefore reducing relative and absolute reliability. It is suggested that all 352 researchers test the reliability to eliminate variance in subjectivity before main 353 analysis (assessing whether impact point affects putting direction variability) takes 354 place.

\section{References}

Alessandrini, S. M. (1995). A motivational example for the numerical solution of twopoint boundary-value problems. SIAM review, 37(3), 423-427.

360 Brouillette, M. (2010). Putter features that influence the rolling motion of a golf ball. Procedia Engineering, 2(2), 3223-3229.

362 Brouillette, M., \& Valade, G. (2008). The effect of putter face grooves on the incipient rolling motion of a golf ball. In: Crews, D., \& Lutz, R., editors. Science and golf V: Proceedings of the World Scientific Congress of Golf. Mesa, Arizona: Energy in Motion; pp. 363-368.

366 Cross, R., \& Nathan, A. M. (2007). Experimental study of the gear effect in ball collisions. American Journal of Physics, 75, 658.

368 Dorsel, T. N., \& Rotunda, R. J. (2001). Low scores, top 10 finishes, and big money: An analysis of professional golf association tour statistics and how these relate to overall performance. Perceptual and Motor Skills, 92(2), 575-585. 
371 Hopkins, W. G. (2000). Measures of reliability in sports medicine and science. Sports Medicine, 30(1), 1-15.

373 Hurrion, P. D., \& Hurrion, R. D. (2008). An investigation into weight distribution and kinematic parameters during the putting stroke. In: Crews,D., \& Lutz, R., editors. Science and golf V: Proceedings of the World Scientific Congress of Golf. Mesa, Arizona: Energy in Motion; pp. 223-238.

Joseph, C. W., Bradshaw, E. J., Kemp, J., \& Clark, R. A. (2013). The Interday Reliability of Ankle, Knee, Leg, and Vertical Musculoskeletal Stiffness During Hopping and Overground Running. Journal of Applied Biomechanics, 29(4), 386-394.

Karlsen, J., Smith, G., \& Nilsson, J. (2008). The stroke has only a minor influence on 382 direction consistency in golf putting among elite players. Journal of Sports Sciences, 26(3), 243-250.

Lorensen, W. E., \& Yamrom, B. (1992). Golf green visualization. IEEE Computer 385 Graphics and Applications, 12(4), 35-44.

386 Pelz, D. T. (2000). Dave Pelz's Putting Bible. New York: Random House.

387 Penner, A. R. (2002). The physics of putting. Canadian Journal of Physics, 80(3), 83388 96.

Quinn, R. J. (2006). Exploring correlation coefficients with golf statistics. Teaching Statistics, 28(1), 10-13.

391 Salkind, N. J. (2011). Statistics for People who (Think They) Hate Statistics. Portland: 392 Sage.

393 Saunders, P. U., Telford, R. D., Pyne, D. B., Peltola, E. M., Cunningham, R. B., Gore, 394 C. J., \& Hawley, J. A. (2006). Short-term plyometric training improves running 
economy in highly trained middle and long distance runners. The Journal of Strength \& Conditioning Research, 20(4), 947-954.

397 Wiseman, F., \& Chatterjee, S. (2006). Comprehensive analysis of golf performance 398 on the PGA tour: 1990-2004. Perceptual and Motor Skills, 102(1), 109-117. 
Table 1. Reliability of the impact variable surface area for the combined data set and individual putter-ball combinations.

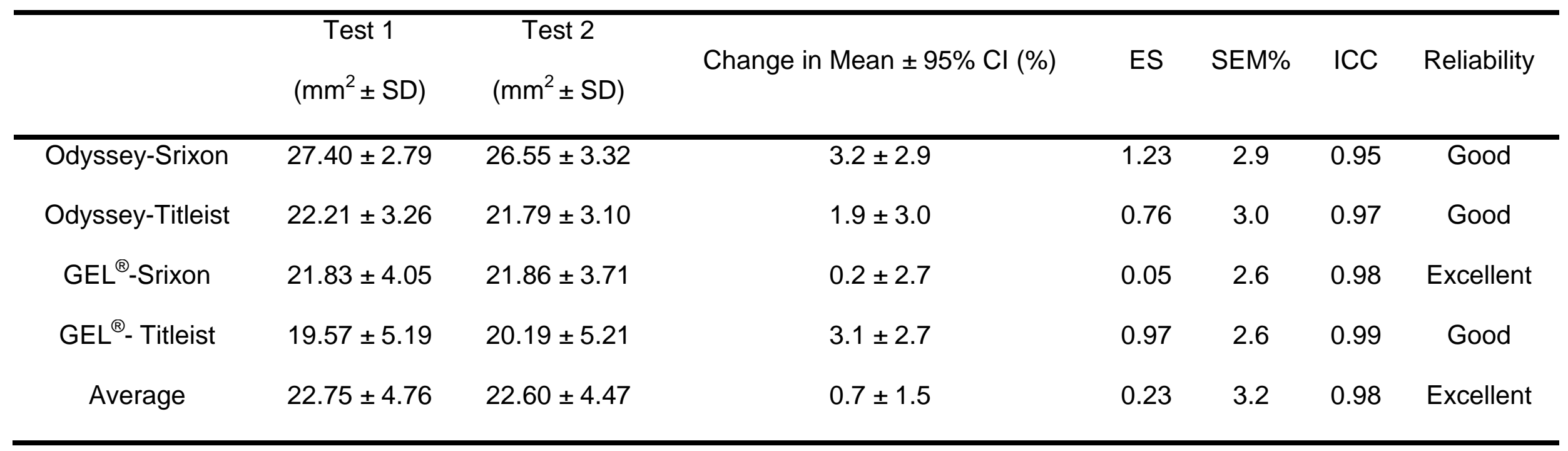

400

401

402

403 

combinations.

\begin{tabular}{cccccccc}
\hline & Test 1 & Test 2 & Change in Mean $\pm 95 \% \mathrm{Cl}(\%)$ & ES & SEM\% & ICC & Reliability \\
& $(\mathrm{mm} \pm \mathrm{SD})$ & $(\mathrm{mm} \pm \mathrm{SD})$ & & 0.43 & 2.6 & 1.00 & Excellent \\
\hline Odyssey-Srixon & $1.56 \pm 0.73$ & $1.57 \pm 0.73$ & $0.6 \pm 2.6$ & 0.00 & 2.1 & 1.00 & Excellent \\
Odyssey-Titleist & $2.86 \pm 0.80$ & $2.86 \pm 0.80$ & $0.0 \pm 2.1$ & 0.55 & 2.9 & 1.00 & Excellent \\
GEL ${ }^{\circledR}$-Srixon & $1.37 \pm 0.57$ & $1.36 \pm 0.59$ & $0.7 \pm 2.9$ & 0.70 & 1.6 & 1.00 & Good \\
GEL ${ }^{\circledR}$-Titleist & $2.51 \pm 0.91$ & $2.53 \pm 0.90$ & $0.8 \pm 1.6$ & 0.00 & 1.9 & 1.00 & Excellent \\
Average & $2.08 \pm 0.97$ & $2.08 \pm 0.97$ & & & & & \\
\hline
\end{tabular}


416 Table 3. Reliability of the impact variable distance derived from the $\mathrm{X}, \mathrm{Y}$ coordinates from the centroid location for the combined

417 data set and individual putter-ball combinations.

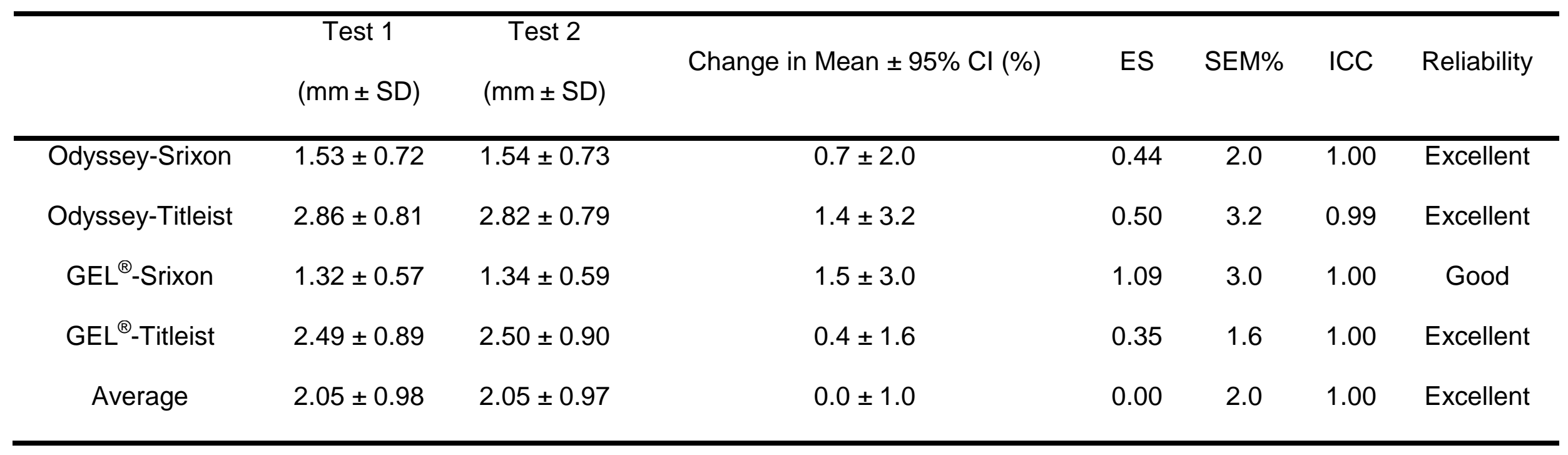

418

419

420

421

422 

combinations.

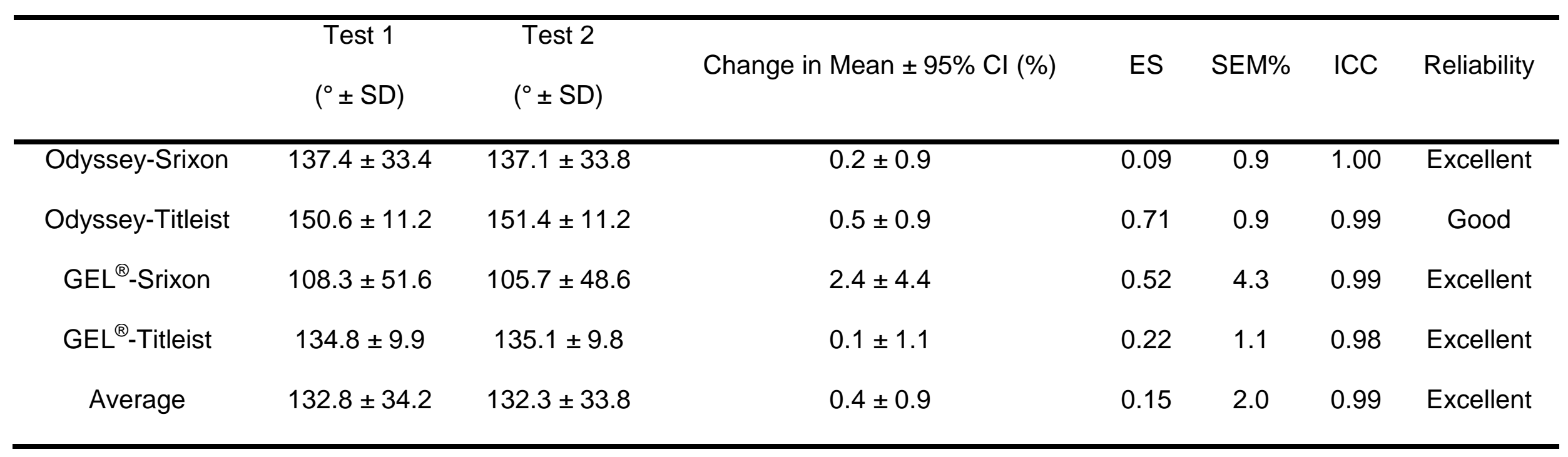



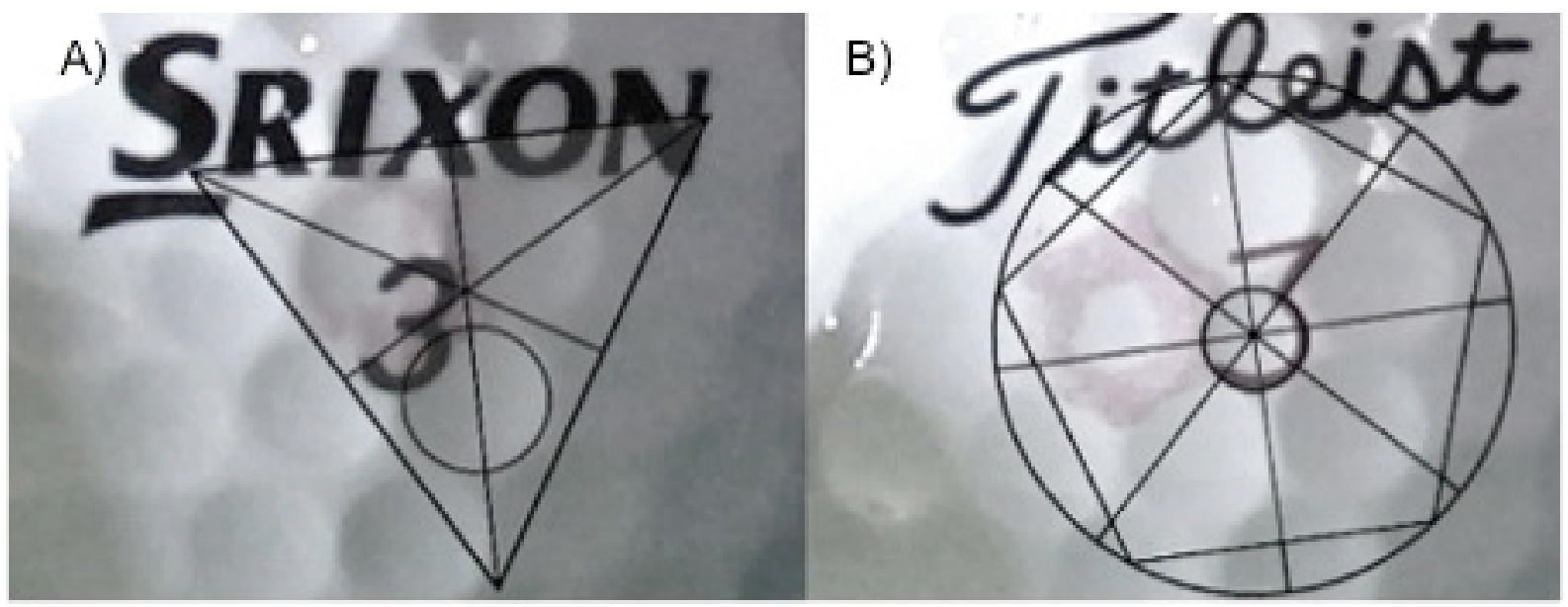

428 Figure 1. Two polygon structures developed to identify the centroid of the A) Srixon 429 and B) Titleist golf ball.

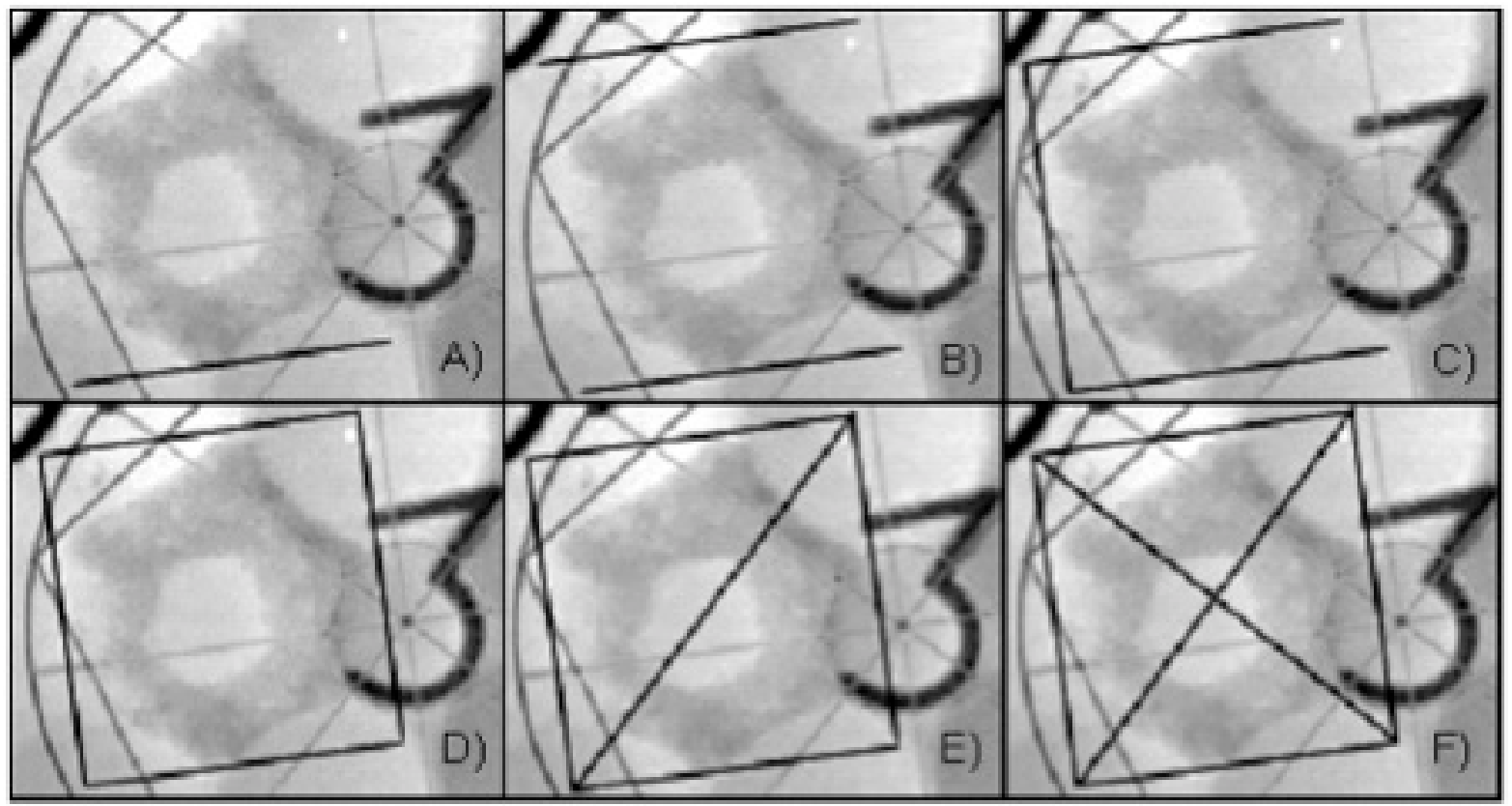

431 Figure 2. Step by step process of constructing and intersecting a polygon to identify 432 the coordinate of the impact point.

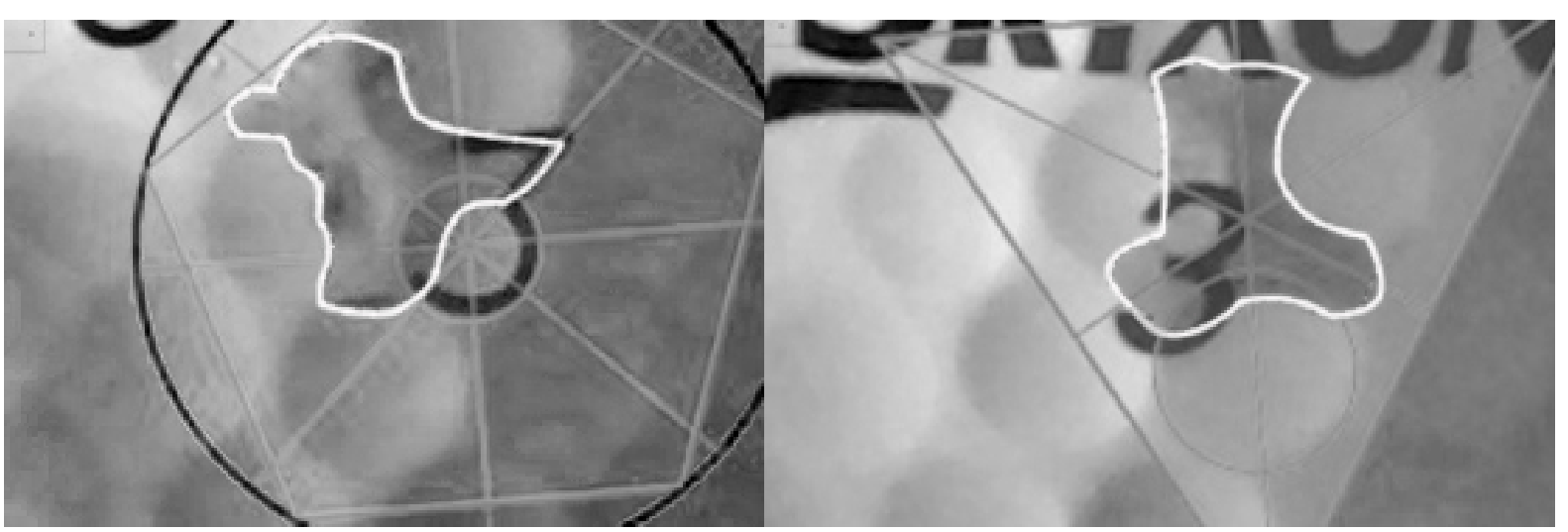


434 Figure 3. Titleist and Srixon golf balls with the polygon outline for calculation of the 435 impact area.

436 